\title{
Simulation of Double-Null Divertor Plasmas with the UEDGE Code
}

M. E. Rensink, S. L. Allen, G. D. Porter, T. D. Rognlien

This article was submitted to

$7^{\text {th }}$ International Workshop on Plasma Edge Theory in Fusion Devices, Tajimi, Gifu, Japan, October 4-6, 1999

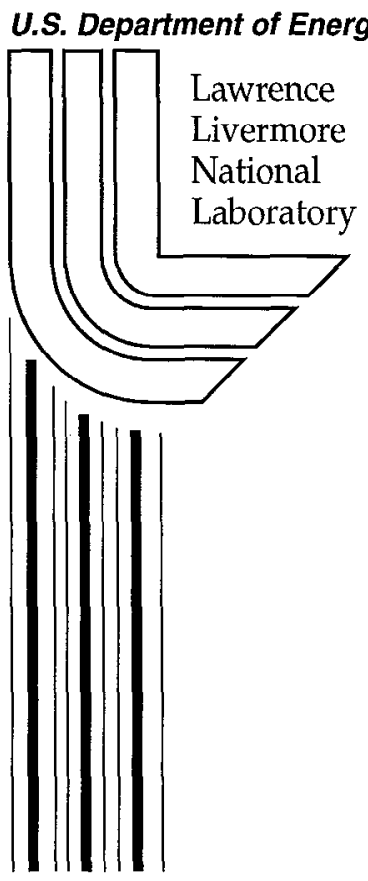

\section{September 21, 1999}




\section{DISCLAIMER}

This document was prepared as an account of work sponsored by an agency of the United States Government. Neither the United States Government nor the University of California nor any of their employees, makes any warranty, express or implied, or assumes any legal liability or responsibility for the accuracy, completeness, or usefulness of any information, apparatus, product, or process disclosed, or represents that its use would not infringe privately owned rights. Reference herein to any specific commercial product, process, or service by trade name, trademark, manufacturer, or otherwise, does not necessarily constitute or imply its endorsement, recommendation, or favoring by the United States Government or the University of California. The views and opinions of authors expressed herein do not necessarily state or reflect those of the United States Government or the University of California, and shall not be used for advertising or product endorsement purposes.

This is a preprint of a paper intended for publication in a journal or proceedings. Since changes may be made before publication, this preprint is made available with the understanding that it will not be cited or reproduced without the permission of the author.

This report has been reproduced directly from the best available copy.

Available to DOE and DOE contractors from the Office of Scientific and Technical Information

P.O. Box 62, Oak Ridge, TN 37831

Prices available from (423) 576-8401

http:/ /apollo.osti.gov/bridge/

Available to the public from the National Technical Information Service

U.S. Department of Commerce 5285 Port Royal Rd., Springfield, VA 22161 http://www.ntis.gov/

OR

Lawrence Livermore National Laboratory Technical Information Department's Digital Library http://www.llnl.gov/tid/Library.html 


\title{
Simulation of Double-Null Divertor Plasmas with the UEDGE Code
}

\author{
M. E. Rensink, S. L. Allen(a), G. D. Porter, T. D. Rognlien \\ Lawrence Livermore National Laboratory, Livermore, CA 94550, USA \\ (a) also at General Atomics, San Diego, CA 92186, USA \\ e-ınail: rensink1@llul.gov
}

\begin{abstract}
We describe recent modifications to the UEDGE code that enable us to simulate full double-null magnetic configurations. We present simulation results for magnetically balanced double nulls, with and without asymmetries due to divertor recycling and cross-field drifts. Up/down electron temperature asymmetries due to recycling are weak for attached plasmas because the magnetic connection length is shorter than for comparable single-null plasmas. Cross-field drifts due to radial electric fields produce strong poloidal ion particle huxes at the midplane in the SOL that can drive up/down asymmetries.
\end{abstract}

\section{Introduction}

Peak heat flux is a limiting factor in divertors for tokamak reactors. Some reduction in peak heat flux can be achieved by splitting the divertor power via double-null configurations. These configurations can also provide increased flexibility for power and particle control in present experiments, but they complicate the interpretation of observed plasma asymmetries. In DIII-D double-null discharge experiments [1] the plasma asymmetries can be attributed to a combination of magnetic imbalance, divertor plate and pumping configuration asymmetries and cross-field drifts. Electrical biasing of the divertor plates can also induce asymmetries. In this report we begin to assess the relative importance of these effects with simulation results from the UEDGE code [2]. Previous work by Marchand [3] addressed the magnetic imbalance issue, but without drifts. For single-null plasmas, UEDGE simulations [4] show that cross-field drifts can significantly affect the inboard/outboard divertor asymmetry. Up/down asymmetries in double-null configurations are similarly affected.

\section{Model}

The UEDGE code is a two-dimensional fluid model for simulating edge plasmas. Previous versions of the code modeled only the lower half of a double null configuration, and imposed up/down symmetry conditions at the midplane. To simulate a full double null with UEDGE, we have modified the grid generation package so as to produce a computational mesh that includes both $\mathrm{x}$-points. We start by generating separate meshes for the upper and lower halves using the existing capability of the grid generator package. These two halves are mapped into the final structured mesh of the full double null. We impose constraints on the mesh generation to ensure that the same set of flux surfaces are used for both upper and lower halves of the mesh; however, upper and lower private flux regions need not use the same flux surfaces. An example of a balanced double null mesh is shown in Fig. 1

The computational mesh for the UEDGE code consists of quadrilateral cells which are indexed into a logically rectangular array. The indexing of the cells for a full asymmetric double null configuration is illustrated in Fig. 2. It is a generalization of the indexing 


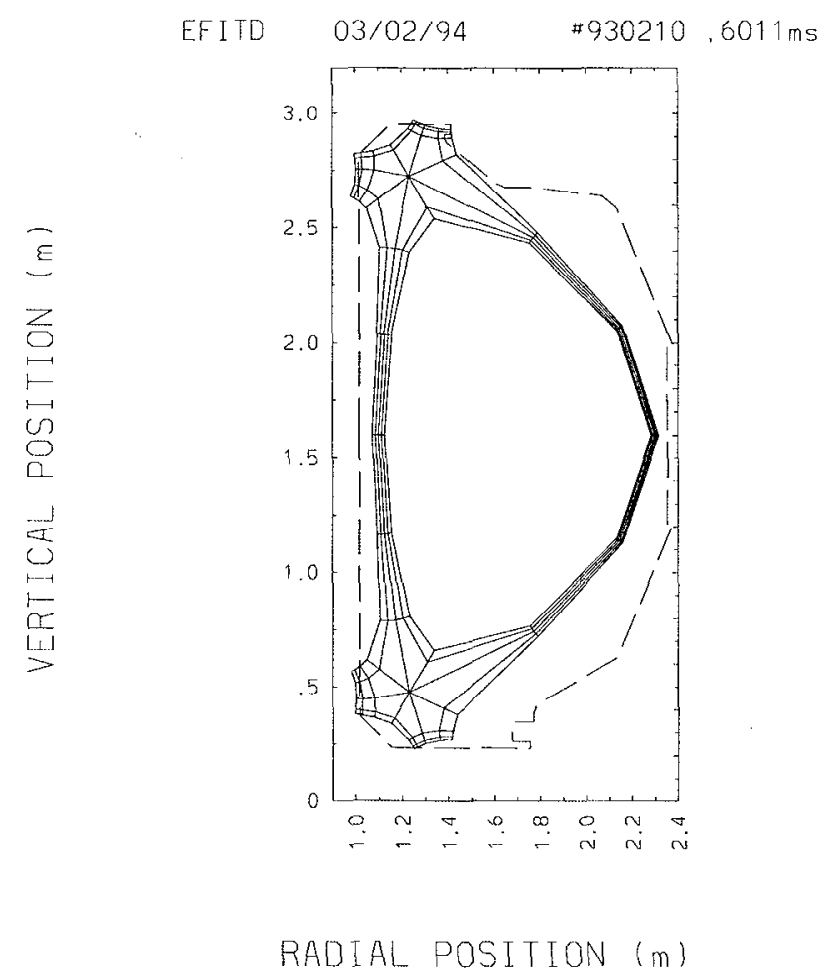

Figure 1: Orthogonal mesh for DIII-D double null. Actual simulations with UEDGE use a finer non-orthogonal mesh that conforms to divertor and vacuum vessel surfaces, indicated by the dashed line.

algorithm for our standard single null configuration. The physical space mesh is separated into two regions by artificial "cuts" extending vertically through the x-points, i.e., the two regions correspond roughly to inboard and outboard halves of the mesh. Each half of the physical mesh is mapped onto a rectangular mesh in the poloidal-radial index space in the standard B2-UEDGE manner.

Inboard and outboard halves of the plasma and target plates are treated in a manner which easily generalizes to multiple x-points. This could allow simulation of toroidally symmetric multipole divertor configurations or stellerator divertor configurations (with assumed helical symmetry). More general x-point configurations could be modelled. For example, a quadrupole configuration in physical space would map to index space as four distinct regions, each of which is similar to the standard B2-UEDGE single null index space.

'The plasma model in UEDGE is essentially unchanged for full double-null simulations. Recycling, pumping and sputtering properties must now be specified separately for each of the distinct surface segments. We do not impose symmetry boundary conditions at the midplane. Continuity conditions at the artificial "cuts" in the mesh are handled automatically by the use of indirect addressing which connects cells on either side of a cut even though they appear in different regions of the mesh in index space. The electrostatic potential is derived from the current flow model described in Ref. [5]. No modification of the potential equation is needed for a double-null configuration, but spatial resolution of sharp gradients near slightly mis-matched separatrices may impose serious practical constraints on the radial mesh. 


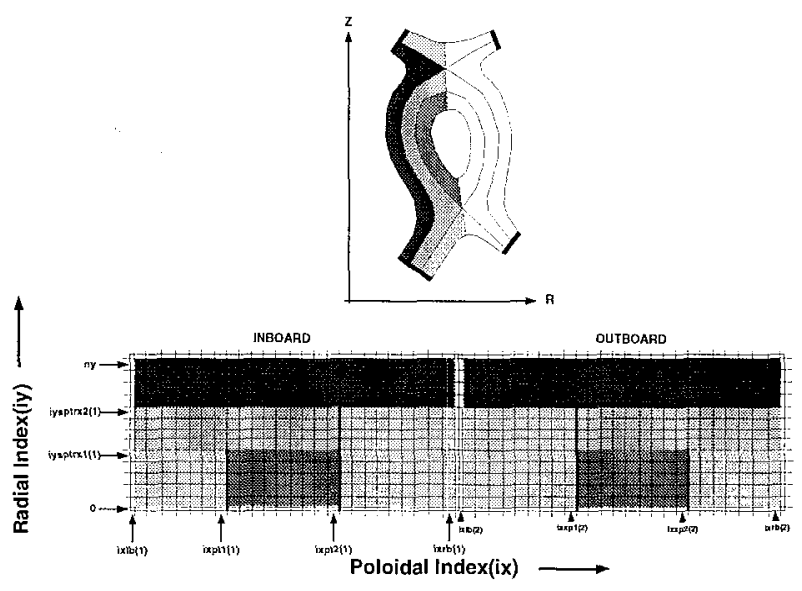

Figure 2: Schematic illustration of mesh in physical space and index space.

\section{Results}

For up/down symmetric mesh and target plate configurations, we find that in the absence of cross field drifts the plasma solutions are exactly symmetric as expected. This demonstrates that our numerical solution algorithm does not introduce artificial asymmetry. This is particularly important because we use a staggered velocity mesh which requires extra care in implementing the numerical differencing, especially near the x-points and target plates. We have successfully tested up/down symmetry for several magnetic geometries, including NSTX [6] and DIII-D. These tests included non-orthogonal meshes, inertial neutrals and electrical current flow to ensure that implementation of these physics models in UEDGE does not contain any built-in asymmetry.

One of the simplest sources of plasma asymmetry can arise due to differences in recycling at the various divertor plates. Sample results that illustrate this effect are shown in Fig. 3. Plasma conditions on the core boundary were fixed density $\left(n=3.5 \times 10^{19} \mathrm{~m}^{-3}\right)$ and temperature $\left(T_{e}=T_{i}=175 \mathrm{eV}\right)$. The ion particle recycling coefficient, $R_{p}$, is defined by

$$
\Gamma_{n}=-R_{p} \cdot \Gamma_{i}
$$

where $\Gamma_{i}$ is the ion particle flux incident on the target plate and $\Gamma_{n}$ is the neutral particle flux reflected or desorbed from the target plate. Symmetric reference cases with particle recycling coefficients $R_{p}=1.0$ and $R_{p}=0.95$ at all target plates show the expected higher plasma temperature with lower recycling. The asymmetric recycling case had $R_{p}=1.0$ on the lower inboard and upper outboard divertor plates with $R_{p}=0.95$ on the upper inboard and lower outboard plates. Two observations are evident: (1) the most significant effect of reducing the recycling on any plate is an overall increase in the level of the electron temperature along the entire flux surface, and (2) the effect of recycling asymmetry is stronger on the outboard flux surfaces where the connection length between plates is longer. The flux surface on the inboard side shows essentially no asymmetry in the electron temperature.

Cross-ficld drifts can give rise to strong inboard/outboard asymmetrics for single null plasmas [4], [5]. In double null configurations they contribute to both inboard/outboard and up/down asymmetry for double nulls. In Fig. 4 we illustrate the effect of drifts on ion particle fluxes across the cuts in the private flux regions and at the midplane for a magnctically balanced configuration in DIII-D. The dircction of the toroidal magnctic field is the "standard" DIII-D configuration, so the ion $\nabla B$-drift is toward the lower x-point. The drifts are calculated for a fixed plasma background, but they are indicative of the changes we 


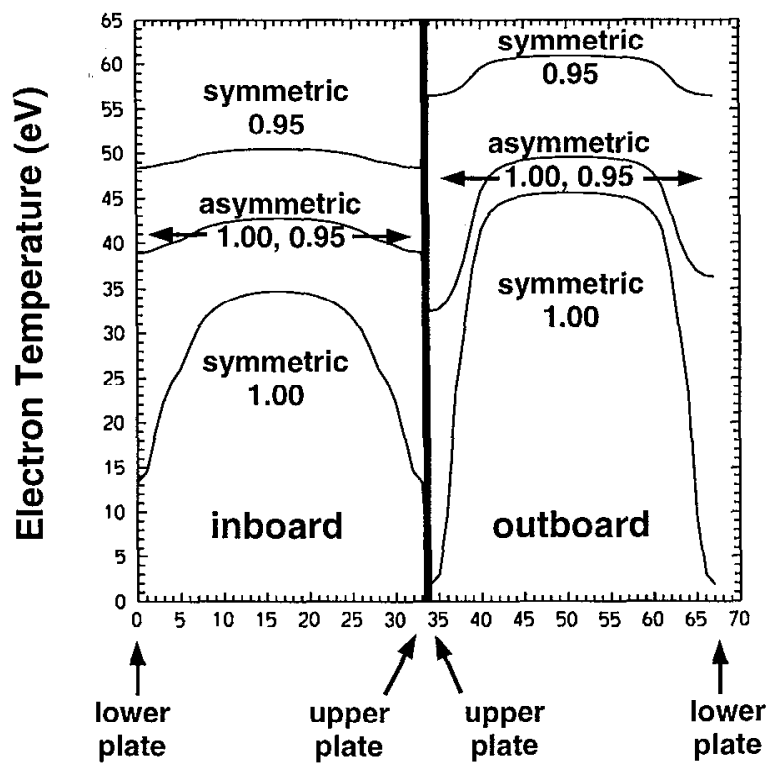

Poloidal Index (ix)

Figure 3: Poloidal variation of electron temperature on a flux surface for various target plate recycling conditions.

can expect in the self-consistent steady state. The dominant effect is due to radial electric fields in the SOL and private flux regions. Near the lower x-point, the electric field in the private flux region is directed downward, giving rise to a particle flux from right to left; near the upper x-point, the electric field in the private flux region is directed upward, so the particle flux is from left to right. At the midplanes, the electric field in the SOL is directed radially outward from the separatrix, so the $\mathbf{E} \times \mathbf{B}$ flux is upward at the inboard midplane and downward at the outboard midplane. These poloidal fluxes are all large compared to the corresponding fluxes in the absence of drifts, so we can expect the ion density in the divertors to change appreciably. One view of these flows is as a net transport of ions from the outboard half to the inboard half, and from the upper half to the lower half. These flows can also be viewed as a net transport ions into and out of the various divertor legs; in this case the lower outboard divertor has the largest net influx and the upper outboard divertor has the largest net outflux. The ion particle fluxes onto the divertor plates are only weakly affected by the drifts. We have not yet obtained a self-consistent steady state for this double null plasma with drifts due to numerical convergence problems. There is considerable structure in the electric field near the separatrix that needs to be resolved.

\section{Summary and Conclusions}

We have shown that the UEDGE code can simulate full double null edge plasma configurations. Up/down asymmetries due to divertor recycling and cross-field drifts have been demonstrated for magnetically balanced double nulls. 


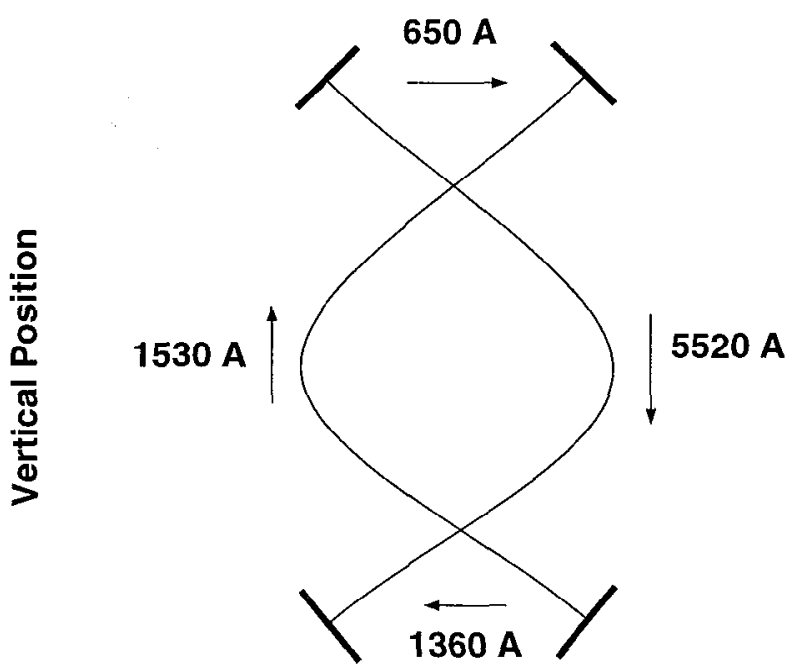

Radial Position

Figure 4: Total ion particle flux at the midplane and under the x-points, mainly due to $\mathbf{E} \times \mathbf{B}$ drifts. Totals do not include flows inside the separatrix.

\section{Acknowledgements}

This work was performed under the auspices of the United States Department of Energy by the Lawrence Livermore National Laboratory under contract number W-7405-ENG-48 and by General Atomics under contract number DE-AC03-89ER51114.

\section{References}

[1] Allen, S.L., et al., J. Nucl. Mater. 266-269 (1999) 168

[2] Rognlien, T.D., et al., Contr. Plasma Phys. 34 (1994) 362

[3] Marchand, R., et al. Nuclear Fusion 35 (1995) 297

[4] Rognlien, T.D., et al., J. Nucl. Mater. 266-269 (1999) 654

[5] Rognlien, T.D., et al., Phys. Plasmas 6 (1999) 1851

[6] NSTX reference? 